\title{
,ance \\ A Machine Learning Ensemble Based on Radiomics to Predict BI-RADS Category and Reduce the Biopsy Rate of Ultrasound-Detected Suspicious Breast Masses
}

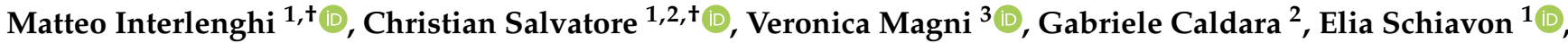

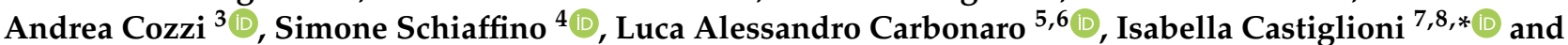 \\ Francesco Sardanelli ${ }^{3,4}$ [D
}

check for updates

Citation: Interlenghi, M.; Salvatore, C.; Magni, V.; Caldara, G.; Schiavon,

E.; Cozzi, A.; Schiaffino, S.;

Carbonaro, L.A.; Castiglioni, I.;

Sardanelli, F. A Machine Learning Ensemble Based on Radiomics to Predict BI-RADS Category and Reduce the Biopsy Rate of

Ultrasound-Detected Suspicious Breast Masses. Diagnostics 2022, 12 187. https://doi.org/10.3390/ diagnostics12010187

Academic Editor: Dechang Chen

Received: 2 December 2021

Accepted: 10 January 2022

Published: 13 January 2022

Publisher's Note: MDPI stays neutral with regard to jurisdictional claims in published maps and institutional affiliations.

Copyright: (C) 2022 by the authors. Licensee MDPI, Basel, Switzerland. This article is an open access article distributed under the terms and conditions of the Creative Commons Attribution (CC BY) license (https:// creativecommons.org/licenses/by/ $4.0 /)$.
1 DeepTrace Technologies S.R.L., Via Conservatorio 17, 20122 Milano, Italy; interlenghi@deeptracetech.com (M.I.); salvatore@deeptracetech.com (C.S.); schiavon@deeptracetech.com (E.S.)

2 Department of Science, Technology and Society, Scuola Universitaria IUSS, Istituto Universitario di Studi Superiori, Piazza della Vittoria 15, 27100 Pavia, Italy; gabriele.caldara@iusspavia.it

3 Department of Biomedical Sciences for Health, Università Degli Studi di Milano, Via Luigi Mangiagalli 31, 20133 Milano, Italy; veronica.magni@unimi.it (V.M.); andrea.cozzi1@unimi.it (A.C.); francesco.sardanelli@unimi.it (F.S.)

4 Unit of Radiology, IRCCS Policlinico San Donato, Via Rodolfo Morandi 30, 20097 San Donato Milanese, Italy; simone.schiaffino@grupposandonato.it

5 Department of Radiology, ASST Grande Ospedale Metropolitano Niguarda, Piazza dell'Ospedale Maggiore 3, 20162 Milano, Italy; luca.carbonaro@unimi.it

6 Department of Oncology and Hemato-Oncology, Università degli Studi di Milano, Via Festa del Perdono 7, 20122 Milan, Italy

7 Institute of Biomedical Imaging and Physiology, Consiglio Nazionale delle Ricerche, Via Fratelli Cervi 93, 20090 Segrate, Italy

8 Department of Physics, Università degli Studi di Milano-Bicocca, Piazza Della Scienza 3, 20126 Milano, Italy

* Correspondence: isabella.castiglioni@unimib.it

+ These authors contributed equally to this work.

\begin{abstract}
We developed a machine learning model based on radiomics to predict the BI-RADS category of ultrasound-detected suspicious breast lesions and support medical decision-making towards short-interval follow-up versus tissue sampling. From a retrospective 2015-2019 series of ultrasound-guided core needle biopsies performed by four board-certified breast radiologists using six ultrasound systems from three vendors, we collected 821 images of 834 suspicious breast masses from 819 patients, 404 malignant and 430 benign according to histopathology. A balanced image set of biopsy-proven benign $(n=299)$ and malignant $(n=299)$ lesions was used for training and cross-validation of ensembles of machine learning algorithms supervised during learning by histopathological diagnosis as a reference standard. Based on a majority vote (over $80 \%$ of the votes to have a valid prediction of benign lesion), an ensemble of support vector machines showed an ability to reduce the biopsy rate of benign lesions by $15 \%$ to $18 \%$, always keeping a sensitivity over $94 \%$, when externally tested on 236 images from two image sets: (1) 123 lesions (51 malignant and 72 benign) obtained from two ultrasound systems used for training and from a different one, resulting in a positive predictive value (PPV) of 45.9\% (95\% confidence interval 36.3-55.7\%) versus a radiologists' PPV of $41.5 \%$ ( $p<0.005)$, combined with a 98.0\% sensitivity (89.6-99.9\%); (2) 113 lesions (54 malignant and 59 benign) obtained from two ultrasound systems from vendors different from those used for training, resulting into a 50.5\% PPV (40.4-60.6\%) versus a radiologists' PPV of $47.8 \%(p<0.005)$, combined with a $94.4 \%$ sensitivity $(84.6-98.8 \%)$. Errors in BI-RADS 3 category (i.e., assigned by the model as BI-RADS 4 ) were $0.8 \%$ and $2.7 \%$ in the Testing set $I$ and $I I$, respectively. The boardcertified breast radiologist accepted the BI-RADS classes assigned by the model in 114 masses $(92.7 \%)$ and modified the BI-RADS classes of 9 breast masses $(7.3 \%)$. In six of nine cases, the model performed better than the radiologist did, since it assigned a BI-RADS 3 classification to histopathology-confirmed benign masses that were classified as BI-RADS 4 by the radiologist.
\end{abstract}


Keywords: breast cancer; ultrasound (US); core needle biopsy; machine learning; radiomics; sensitivity; positive predictive value

\section{Introduction}

Ultrasound imaging is a key tool in breast care. Indications to breast ultrasound, recently summarized by the European Society of Breast Imaging (EUSOBI) [1], include palpable lump; axillary adenopathy; first approach for clinical abnormalities in women younger than 40 years of age and in pregnant or lactating women; suspicious abnormalities revealed at mammography or contrast-enhanced magnetic resonance imaging (MRI); suspicious nipple discharge; skin retraction; recent nipple inversion; breast inflammation; abnormalities at the site of intervention after breast-conserving surgery or mastectomy; abnormalities in the presence of oncoplastic or aesthetic breast implants. Moreover, when MRI is not performed, the following indications to breast ultrasound can be considered: screening high-risk women or women with extremely dense breasts (supplemental to mammography); loco-regional staging of a known breast cancer; monitoring breast cancers receiving neoadjuvant systemic therapy. In addition, ultrasound provides an optimal, cheap, and comfortable guidance for performing needle biopsy for suspicious ultrasounddetected breast lesions, including those initially detected at digital mammography techniques (two-dimensional, tomosynthesis, or contrast-enhanced mammography) or MRI, when a sure correlation between the ultrasound finding and the initially detected finding can be established [2,3].

Indeed, since benign abnormalities (and sometimes also normal breast tissues) are able to mimic malignancies even on advanced breast imaging modalities and techniques, tissue sampling represents the best method for confirmation or exclusion of breast cancer [2,3]. Thus, in the last decades, percutaneous needle biopsy has been established as a crucial approach to prevent unnecessary surgery, and reduce associated morbidity as well as economic and psychological costs associated with suspicious findings finally being demonstrated to be benign. The European Society of Breast Cancer Specialists (EUSOMA) includes, among the mandatory quality indicators in breast cancer care [4], the assessment of the "proportion of women with breast cancer (invasive or in situ) who had a preoperative histologically or cytologically confirmed malignant diagnosis (B5 or C5)". For this indicator, EUSOMA requires a "minimum standard" rate of $85 \%$ and a target rate of $90 \%$ [4]. The dark side of the moon of the worldwide practice of percutaneous breast needle biopsy, mostly performed under ultrasound guidance, is the variable and frequently high rate of procedures needed to exclude malignancy for findings that finally are revealed to be benign. To avoid missing cancers, breast radiologists are "forced" to biopsy also many abnormalities with probably benign features, unless they think that a given lesion in a given patient, also considering patient-specific risk factors (family and personal history as well as clinical conditions), has an extremely low probability of being malignant and that a six-month delayed diagnosis will not impact on patient's outcome. Using the Breast Imaging Reporting and Data System (BI-RADS), this means to categorize the lesion as BI-RADS 3, which should imply a residual cancer probability lower than $2 \%$, against a cancer probability higher than $2 \%$ but lower than $95 \%$ (BI-RADS 4 ) and a cancer probability higher than 95\% (BI-RADS 5) [5]. New approaches aiming at reducing the ultrasoundguided biopsy rate of benign breast lesions must take into account such a challenging clinical context.

Machine learning is a methodological approach of artificial intelligence that concerns building systems that learn based on the data they use. It is widely used in medical imaging to develop image-driven multivariate systems effective in complex tasks, such as supporting physicians in clinical decision-making [6]. Radiomics, i.e., the measurement of a high number of quantitative features from images characterizing size, shape, image intensity, and texture of identified findings, has been extensively used to train multivariate 
machine learning algorithms to objectively characterize image findings and to predict diagnosis and prognosis of individual lesions or subjects. In breast cancer care, radiomics has been applied to a variety of medical image modalities for the aforementioned purposes, including mammography, digital breast tomosynthesis, ultrasound, magnetic resonance imaging, and positron-emission tomography combined with computed tomography [7-10], with good performances and with the advantage of high explainability, in particular when the radiomic predictors of the models can be compared and interpreted with reference to semantic predictors previously described in literature. In particular, many features of breast lesions on ultrasound images are known to be associated with higher or lower probability of malignancy of a given lesion, as Stavros et al. [11] pointed out in their seminal paper focused on breast solid masses published more than 25 years ago. These authors described traditional features such as shape, margins, spatial orientation, absolute signal intensity, signal intensity relative to the surrounding tissue (the classic hyper-, iso-, and hypoechoic patterns), and signal heterogeneity, all of them integrated in the BI-RADS lexicon [5]. However, it is difficult for a human reader to attain quantification and integration of such a wide spectrum of information, whereas it is expected to be best achieved through a multivariate model of radiomics and machine learning.

Therefore, the aim of our study was to develop and validate a machine learning model based on radiomics to classify ultrasound-detected suspicious breast masses with the specific two-fold purpose of providing a second opinion on BI-RADS classification and of reducing the needle biopsy rate. A high sensitivity combined with a sizable reduction in the number of false positive cases were the guiding criteria to develop the machine learning model. The best radiomic predictors were specifically described and interpreted to explain the model and its results.

\section{Materials and Methods}

This study retrospectively analyzed the breast biopsy database of the Radiology Unit at IRCCS Policlinico San Donato (San Donato Milanese, Milan, Italy) and was approved by the institutional ethics committee (Comitato Etico IRCCS Ospedale San Raffaele, protocol code "SenoRetro", first approved on 9 November 2017, then amended on 18 July 2019, and on 12 May 2021). The acquisition of specific informed consent was waived due the retrospective nature of the study.

\subsection{Study Population and Image Sets}

A consecutive series of 926 patients referred for ultrasound-guided core needle biopsy from 13 January 2014, to 28 May 2019 was retrieved, for a total of 928 ultrasound images of 941 suspicious breast masses according to the judgment of one of four rotating certified breast radiologists with 4 to 14 years of experience in breast imaging. All ultrasound images were acquired with one of six ultrasound systems (Esaote MyLab 6100, MyLab 6150, MyLab 6440, and MyLab 7340002, Esaote S.p.A, Genova, Italy; Samsung RS80A, Samsung Healthcare, Seoul, South Korea; Acuson Juniper, Siemens Healthineers, Erlangen, Germany). After database search, another certified breast radiologist with 34 years of experience in breast imaging retrospectively reviewed all images to identify the biopsied lesion on the ultrasound images, excluding 96 images from 96 women for which a sure identification of the biopsied mass was not attainable. Ultimately, 821 ultrasound images of 834 suspicious breast masses from 819 patients (mean age $56 \pm 16$ (standard deviation) years) were considered for radiomic analysis and to develop and test the machine learning model. Histopathology from core needle biopsy or pathology of surgical specimens was used as a reference standard, with $404 / 834$ lesions (48.4\%) proven to be malignant and 430/834 lesions (51.6\%) proven to be benign, for an overall 1.06:1.00 benignto-malignant ratio.

A balanced set of randomly sampled ultrasound images from 299 malignant and 299 benign lesions, all from three of the six ultrasound systems (Esaote MyLab 6100, MyLab 6150, MyLab 6440, and MyLab 7340002), were used for the training and internal testing of 
different ensembles of machine learning classifiers, based on the supervised learning of histopathology as a reference standard (Training and internal testing set). Then, the remaining images of 123 other lesions (51 malignant and 72 benign according to histopathology), obtained from two of the ultrasound systems of the Training and internal testing set and from a third one, were used as first external testing for the best machine learning model (Testing set I). Finally, the remaining images of the 113 lesions ( 54 malignant and 59 benign according to histopathology), obtained from the other two of the six considered ultrasound systems (Samsung RS80A and Siemens Healthineers Acuson Juniper), were used as second external testing for the best machine learning model (Testing set II).

\subsection{Radiomic-Based Machine Learning Modelling}

Radiomic methodology was applied to the 821 included images, according to the International Biomarker Standardization Initiative (IBSI) guidelines [12]. For this purpose, the TRACE4@ radiomic platform [13] was used, allowing the whole IBSI-compliant radiomic workflow to be obtained in a fully automated way. The IBSI radiomic workflow included (i) segmentation of the suspicious mass to obtain a region of interest (ROI) from each patient image; (ii) preprocessing of image intensities within the segmented ROI required to measure radiomic features; (iii) measurement of radiomic features from the segmented ROI; (iv) the use of such candidate radiomic features to train, validate, and test different models of machine learning classifiers in the binary classification task of interest (malignant versus benign discrimination), by the reduction of such extracted features to reliable and nonredundant features.

More specifically, the workflow in this study was as follows:

1. The segmentation of suspicious masses on all 821 images was performed manually by a board-certified radiologist with 34 years of experience in breast imaging, using the TRACE4 segmentation tool. The same radiologist (at a time distance of 8 weeks) and a second board-certified radiologist with 7 years of experience independently segmented the masses on a random subsample of 50 images from the training dataset, fully blinded to histopathology and other segmentations.

2. The preprocessing of image intensities within the segmented ROI included resampling to isotropic voxel spacing, using a downsampling scheme by considering an image slice thickness equal to pixel spacing, and intensity discretization using a fixed number of 64 bins.

3. The radiomics features measured from the segmented ROI were 107 quantitative descriptors and belonged to different families: morphology, intensity-based statistics, intensity histogram, grey-level co-occurrence matrix (GLCM), grey-level run length matrix (GLRLM), grey-level size zone matrix (GLSZM), neighborhood grey tone difference matrix (NGTDM), grey-level distance zone matrix (GLDZM), and neighboring grey-level dependence matrix (NGLDM). Their definition, computation, and nomenclature are compliant with the IBSI guidelines, except for the features of the family morphology, originally designed for 3D images, which were replaced with ten 2D equivalent features (e.g., 3D features volume and surface were replaced with 2D features area and perimeter, respectively). Radiomic features were selected as those showing an intraclass correlation coefficient $>0.75$ among the two intra-observer and inter-observer segmentations on the random subsample of images described in point (1), since according to the $95 \%$ confidence interval of the intraclass correlation coefficient estimate, values lower than 0.5 , between 0.5 and 0.75 , between 0.75 and 0.9 , and higher than 0.9 are indicative of poor, moderate, good, and excellent reliability, respectively [14]. Steps from (2) to (3) were performed using the TRACE4 Radiomics tool. Radiomic features were reported by TRACE4 according to IBSI standards.

4. Three different models of machine learning classifiers were trained, validated, and tested, for the binary classification task of interest (malignant versus benign discrimination), based on supervised learning, using histopathology as a reference standard. For each model, a nested $k$-fold cross-validation method was used $(k=10,8$ folds 
for training, 1 fold for tuning, 1 fold for hold-out testing, random sampling). The first model consisted of 3 ensembles of 100 random forest classifiers combined with Gini index with majority-vote rule; the second model consisted of 3 ensembles of 100 support vector machines (linear kernel) combined with principal components analysis and Fisher discriminant ratio with majority-vote rule; the third ensemble consisted of 3 ensembles of 100 k-nearest neighbor classifiers combined with principal components analysis and Fisher discriminant ratio with majority-vote rule. Data for the ensemble learning set were selected by using 100 baggings based on random sampling without replacement ( $80 \%$ data for training, $10 \%$ data for tuning, $10 \%$ data for internal testing). Each classifier belonging to the same ensemble was tested internally on datasets that can not have data samples in common. Classifiers belonging to different ensembles were tested on datasets that can have samples in common. The performances of the 3 models were measured across the different folds $(k=10)$ in terms of sensitivity, specificity, area under the receiver operating characteristic curve (ROC-AUC), positive predictive value (PPV), negative predictive value (NPV), and corresponding $95 \%$ confidence intervals $(\mathrm{CI})$. The model with the best performance according to ROC-AUC was chosen as the best classification model for the binary task of interest (malignant versus benign discrimination).

For the best classification model, a study of the percentage of the votes of the classifiers in an ensemble to have a valid prediction (concordance on predicted class higher than a qualified majority) of benign and malignant lesions was performed during cross-validation in order to maximize sensitivity. Ultimately, this machine learning model was tested on the two external datasets (Testing set I and Testing set II).

Relevant radiomic predictors were selected as those radiomic features most frequently chosen by the machine learning classifiers as the most relevant ones during the crossvalidation of the ensembles. For random forest classifiers, the mean importance of each radiomic feature was obtained by each random forest classifier during validation on outof-bag samples. For support vector machines and k-nearest neighbor classifiers, the mean weight coefficient of each radiomic feature was obtained as explained by each principal component selected by the classifier through a grid search on validation samples.

\subsection{BI-RADS Diagnostic Categories Classification}

When the percentage of the votes of the classifiers in the best ensemble had a valid prediction of benign lesions (concordance on predicted benign class higher than the qualified majority), the ensemble assigned the BI-RADS 3 category. Similarly, when the percentage of the votes of the classifiers in the best ensemble had a valid prediction of malignant lesions, the ensemble assigned the BI-RADS 4 or 5 category according to the level of concordance of the majority of support vectors in the ensemble. For each of the breast masses of the Testing set I (123 masses), the certified breast radiologist with 34 years of experience in breast imaging accepted or modified the BI-RADS category assigned by the best ensemble (best model), blinded to histopathology. The class agreement and disagreement were assessed on a case-by-case basis using histopathology as reference standard. Of course, in this assessment, BI-RADS categories 1 (no abnormalities), 2 (benign lesions), 0 (inconclusive examination), and 6 (known malignancy) were not considered due to the design of the study. The class agreement and disagreement of the random subsample of images, resegmented by the board-certified radiologist with 34 years of experience (intra-observer agreement) and by the board-certified radiologist with 7 years of experience (inter-observer agreement), were assessed on a case-by-case basis using the first segmentation of the board-certified radiologist with 34 years of experience as reference standard. For each comparison between reference standard segmentation and the two resegmentations, mean DICE indices were obtained. In addition, for this assessment, BI-RADS categories 1 (no abnormalities), 2 (benign lesions), 0 (inconclusive examination), and 6 (known malignancy) were not considered due to the design of the study. 


\subsection{Statistical Analysis}

Statistical analysis was conducted with embedded tools of the TRACE4 platform. To describe the distribution of each of the most relevant features in the malignant and benign classes, we calculated their medians with 95\% CIs and presented violin plots and boxplots.

A nonparametric univariate Wilcoxon rank-sum test (Mann-Whitney $U$ test [15]) was performed for each of the relevant radiomic predictors to verify its significance in discriminating malignant from benign lesions. To account for multiple comparisons, the $p$-values were adjusted using the Bonferroni-Holm method and the significance levels were set at $0.05\left(^{*}\right)$ and $0.005\left({ }^{* *}\right)[16]$.

\section{Results}

\subsection{Study Population and Image Sets}

Table 1 details the histopathological classification of the 834 suspicious breast lesions included in the study, while Table 2 lists technical information about the acquisition of the 821 ultrasound images that depicted these 834 lesions and their distribution into image sets used for all phases of the machine learning model development. A total of six different ultrasound systems were considered, four from the same vendor, the other two from different vendors, with an overall mean image pixel size ranging from $0.062 \mathrm{~mm}$ to $0.106 \mathrm{~mm}$. The study population comprised 13 males and 806 females, aged $56.0 \pm 16.1$ years (mean \pm standard deviation).

Table 1. Histopathology of the 834 breast masses included in the study.

\begin{tabular}{cccc}
\hline Malignant or Benign & Histopathology Type & Number & Percentage \\
\hline & Fibroadenoma & 146 & $34.0 \%$ \\
& Sclerosing lesions / adenosis & 64 & $14.9 \%$ \\
Normal breast tissue & 38 & $8.8 \%$ \\
& Inflammatory lesions & 36 & $8.4 \%$ \\
Papilloma (no atypia) & 27 & $6.3 \%$ \\
& Cysts, ductal ectasia, or seromas & 37 & $8.6 \%$ \\
& Usual ductal hyperplasia & 17 & $3.9 \%$ \\
& Atypical ductal hyperplasia & 8 & $1.9 \%$ \\
& Fibroadenomatoid changes & 23 & $5.3 \%$ \\
& Other benign findings & 34 & $7.9 \%$ \\
& Total & $\mathbf{4 3 0}$ & $\mathbf{1 0 0 \%}$ \\
\hline & Invasive ductal carcinoma & 304 & $75.2 \%$ \\
& Invasive lobular carcinoma & 42 & $10.4 \%$ \\
Ductal carcinoma in situ & 19 & $4.7 \%$ \\
Malignant & Other malignancies originating & 35 & $8.7 \%$ \\
& from breast tissues & & \\
& Other malignancies (metastases & 4 & $\mathbf{1 0 0 \%}$ \\
\hline from non breast tissues) & Total & 404 & \\
\hline
\end{tabular}

\subsection{Radiomic-Based Machine Learning Modelling}

Since 107 radiomic features were found stable among the two intra-observer and inter-observer segmentations on the random subsample of images, they were calculated (intraclass correlation coefficient range: 0.758-1.000) and used to train (nested k-fold crossvalidation) and externally test the machine learning ensembles.

The ensemble of support vector machines resulted to be the best system for the task of interest, i.e., discrimination between biopsy-proven benign versus malignant lesions, performance comparison for all ensembles being shown in Tables S1-S3. C parameter values of support vector machines were found in the range of $0.0010-0.0183$ (grid search method). A majority vote $>80 \%$ of machines to have a valid prediction of benign lesions and a majority vote $>50 \%$ of machines to have a valid prediction of malignant lesions 
warranted a sensitivity $>94 \%$ during both training and external testing, which is the crucial performance to be warranted for ultrasound examination of suspicious breast lesions (Figure 1), allowing however a reduction of $15 \%-18 \%$ in the number of the needle biopsies that resulted in benign histopathology; this consensus was chosen as a qualified majority vote for the task of interest in this specific clinical context. Interestingly, as depicted in Figure 1, the sensitivity was $>96 \%$ on images from different ultrasound systems but from the same vendor (Training and internal testing set and Testing set I).

Table 2. Technical details and composition of the three image sets.

\begin{tabular}{|c|c|c|c|c|c|c|c|c|}
\hline Dataset & US System & $\begin{array}{c}\text { US } \\
\text { Images }\end{array}$ & $\begin{array}{c}\text { Total } \\
\text { Lesions }\end{array}$ & $\begin{array}{l}\text { Mean Pixel Size } \\
\text { (Range) (mm) }\end{array}$ & $\begin{array}{l}\text { Malignant } \\
\text { Lesions }\end{array}$ & $\begin{array}{l}\text { Mean Pixel Size } \\
\text { (Range) (mm) }\end{array}$ & $\begin{array}{l}\text { Benign } \\
\text { Lesions }\end{array}$ & $\begin{array}{c}\text { Mean Pixel Size } \\
\text { (Range) }(\mathrm{mm})\end{array}$ \\
\hline \multirow{3}{*}{$\begin{array}{l}\text { Training and } \\
\text { internaltesting }\end{array}$} & Esaote MyLab 6100 & 273 & 277 & $\begin{array}{c}0.098 \\
(0.046-0.154)\end{array}$ & 156 & $\begin{array}{c}0.103 \\
(0.046-0.154)\end{array}$ & 121 & $\begin{array}{c}0.092 \\
(0.046-0.139)\end{array}$ \\
\hline & Esaote MyLab 6150 & 311 & 318 & $\begin{array}{c}0.091 \\
(0.046-0.123)\end{array}$ & 142 & $\begin{array}{c}0.095 \\
(0.046-0.123)\end{array}$ & 176 & $\begin{array}{c}0.088 \\
(0.046-0.123)\end{array}$ \\
\hline & Esaote MyLab 6440 & 2 & 3 & $\begin{array}{c}0.068 \\
(0.068-0.068)\end{array}$ & 1 & $\begin{array}{c}0.068 \\
(0.068-0.068)\end{array}$ & 2 & $\begin{array}{c}0.068 \\
(0.068-0.068)\end{array}$ \\
\hline \multirow{3}{*}{ Testing set I } & Esaote MyLab 6100 & 59 & 59 & $\begin{array}{c}0.091 \\
(0.046-0.109)\end{array}$ & 20 & $\begin{array}{c}0.094 \\
(0.062-0.108)\end{array}$ & 39 & $\begin{array}{c}0.090 \\
(0.046-0.108)\end{array}$ \\
\hline & Esaote MyLab 6150 & 63 & 63 & $\begin{array}{c}0.097 \\
(0.048-0.139)\end{array}$ & 31 & $\begin{array}{c}0.101 \\
(0.048-0.139)\end{array}$ & 32 & $\begin{array}{c}0.092 \\
(0.062-0.123)\end{array}$ \\
\hline & Esaote MyLab 7340002 & 1 & 1 & $\begin{array}{c}0.106 \\
(0.106-0.106) \\
\end{array}$ & 0 & - & 1 & $\begin{array}{c}0.106 \\
(0.106-0.106)\end{array}$ \\
\hline \multirow[b]{2}{*}{ Testing set II } & Samsung RS80A & 86 & 86 & $\begin{array}{c}0.065 \\
(0.040-0.110)\end{array}$ & 44 & $\begin{array}{c}0.068 \\
(0.050-0.110)\end{array}$ & 42 & $\begin{array}{c}0.062 \\
(0.040-0.090)\end{array}$ \\
\hline & $\begin{array}{c}\text { Siemens } \\
\text { Healthineers } \\
\text { Acuson Juniper }\end{array}$ & 26 & 27 & $\begin{array}{c}0.067 \\
(0.030-0.080)\end{array}$ & 10 & $\begin{array}{c}0.069 \\
(0.060-0.070)\end{array}$ & 17 & $\begin{array}{c}0.066 \\
(0.030-0.080)\end{array}$ \\
\hline
\end{tabular}

US, ultrasound.

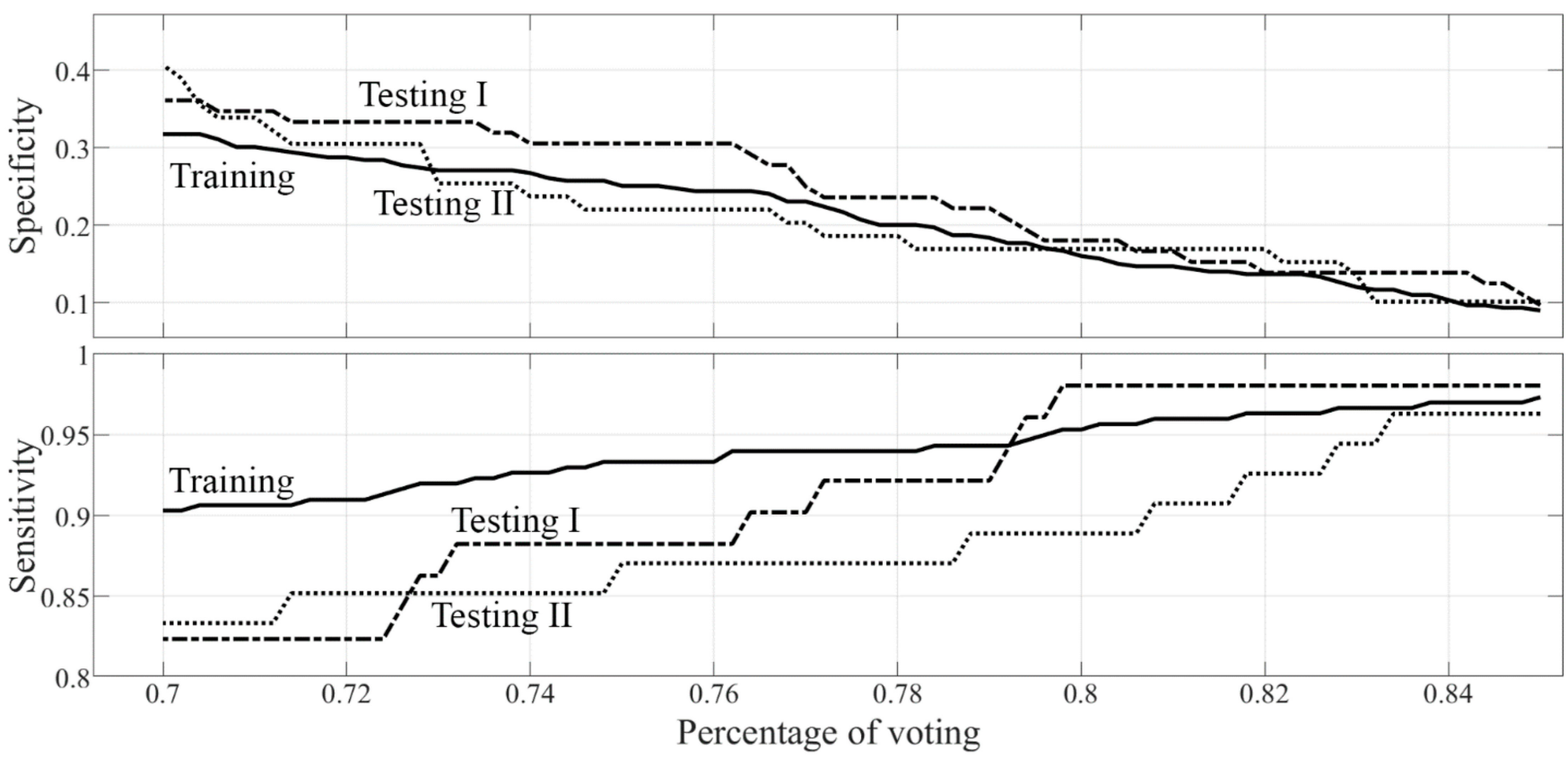

Figure 1. Ensemble of support vector machines: proportion of correctly predicted benign and malignant lesions versus percentage of voting from the support vector machines.

Performance metrics of this high-sensitivity machine learning model in the Training and internal testing set (10-fold cross-validation) were sensitivity 95.7\% ** (95\% CI: 92.7-97.7\%), NPV 78.3\% ** (65.8-87.9\%), PPV 53.2\% ** (48.8-57.4\%), and specificity $15.7 \%$ ** $(11.8-20.3 \%)$. As detailed in Table 3, also presenting comparisons of PPV and specificity with those achieved by the radiologists, performances of the machine learning model in the external Testing set I were sensitivity $98.0 \%$ (89.6-99.9\%), NPV of 92.9\% (66.1-99.8\%), PPV $45.9 \% * *(36.3-55.7 \%)$, and specificity $18.1 * *(10.0-28.9 \%)$. Performances in the external 
Testing set II were sensitivity $94.4 \%$ (84.6-98.8\%), NPV of 75.0\% (42.8-94.5\%), PPV of $50.5 \%$ ** $(40.4-60.6 \%)$, and specificity $15.3 \%$ ** $(7.2-27.0 \%)$.

Table 3. Performances of the ensemble of support vector machines in the external testing datasets.

\begin{tabular}{ccc}
\hline Performance Metric & Testing Set I & Testing Set II \\
\hline SVM sensitivity (95\% CI) & $98.0 \%(89.6 \%-99.9 \%)$ & $94.4 \%(84.6 \%-98.8 \%)$ \\
SVM NPV (95\% CI) & $92.9 \%(66.1 \%-99.8 \%)$ & $75.0 \%(42.8 \%-94.5 \%)$ \\
SVM PPV (95\% CI) & $45.9 \% * *(36.3 \%-55.7 \%)$ & $50.5 \% * *(40.4 \%-60.6 \%)$ \\
Radiologists' PPV & $41.5 \% * *(32.7 \%-50.7 \%)$ & $47.8 \% * *(38.3 \%-57.4)$ \\
SVM specificity (95\% CI) & $18.1 \% * *(10.0 \%-28.9 \%)$ & $15.3 \% * *(7.2 \%-27.0 \%)$ \\
Radiologists' specificity & $0.0 \%$ & $0.0 \%$ \\
\hline
\end{tabular}

SVM, support vector machines; CI, confidence interval; NPV, negative predictive value; PPV, positive predictive value. ${ }^{* *}$ indicates a $p$-value $<0.005$, calculated considering chance/random classification. Note: the $0 \%$ radiologists' specificity is an obliged result determined by the inclusion of breast masses that were all referred to ultrasound-guided core needle biopsy.

Principal components analysis and Fisher discriminant ratio reduced the 107 IBSIradiomic features, measured from each breast lesion of the Training and internal testing set, to an average of 12 (range 7-17) independent principal components for each support vector machine of the ensemble. The top 25 most relevant radiomic predictors selected by such model from the 107 IBSI-compliant features are shown in Table 4, together with their IBSI feature family and feature nomenclature, and ranked according to their frequencies among the most relevant ones in the support vector machines of the ensemble. Results from univariate statistical rank-sum tests are also reported with adjusted $p$-values. The violin plots and boxplots of the first 15 radiomic predictors are shown in Figure 2, while the violin plots and boxplots of the other 10 radiomic predictors are shown in Figure S1.

Table 4. Ensemble of support vector machines. Top 25 most relevant predictors sorted in descending order of relevance.

\begin{tabular}{|c|c|c|}
\hline Rank & Feature Family & $\begin{array}{c}\text { Feature } \\
\text { Name }\end{array}$ \\
\hline 1 & Morphology & Perimeter-to-area ratio ** \\
\hline 2 & Morphology & Maximum diameter ${ }^{* *}$ \\
\hline 3 & Morphology & Compactness ** \\
\hline 4 & Morphology & Acircularity ** \\
\hline 5 & Morphology & Perimeter ** \\
\hline 6 & Morphology & Area ** \\
\hline 7 & Morphology & Center of mass shift ** \\
\hline 8 & Morphology & Circularity* \\
\hline 9 & Neighborhood grey tone difference matrix & Strength ** \\
\hline 10 & Neighborhood grey tone difference matrix & Coarseness ** \\
\hline 11 & Neighborhood grey tone difference matrix & Contrast \\
\hline 12 & Neighborhood grey tone difference matrix & Busyness * \\
\hline 13 & Grey-level size zone matrix & Zone size non-uniformity ** \\
\hline 14 & Grey-level size zone matrix & Grey-level non-uniformity glszm ** \\
\hline 15 & Neighboring grey-level dependence matrix & Dependence count non-uniformity ** \\
\hline 16 & Neighboring grey-level dependence matrix & Low-dependence low-grey-level emphasis \\
\hline 17 & Grey-level run length matrix & Grey-level non-uniformity \\
\hline 18 & Grey-level run length matrix & Run length non-uniformity \\
\hline 19 & Intensity-based statistics & Minimum \\
\hline 20 & Intensity-based statistics & Energy \\
\hline 21 & Intensity-based statistics & Variance \\
\hline 22 & Intensity-based statistics & Quartile coefficient \\
\hline 23 & Intensity-based statistics & 10th percentile \\
\hline 24 & Intensity histogram & 10th percentile \\
\hline 25 & Grey-level co-occurrence matrix & First measure of information correlation \\
\hline
\end{tabular}

${ }^{*}$ denotes statistical significance at 0.05 (adjusted with Bonferroni-Holm correction). ${ }^{* *}$ denotes a statistical significance at 0.005 (adjusted with Bonferroni-Holm correction). 

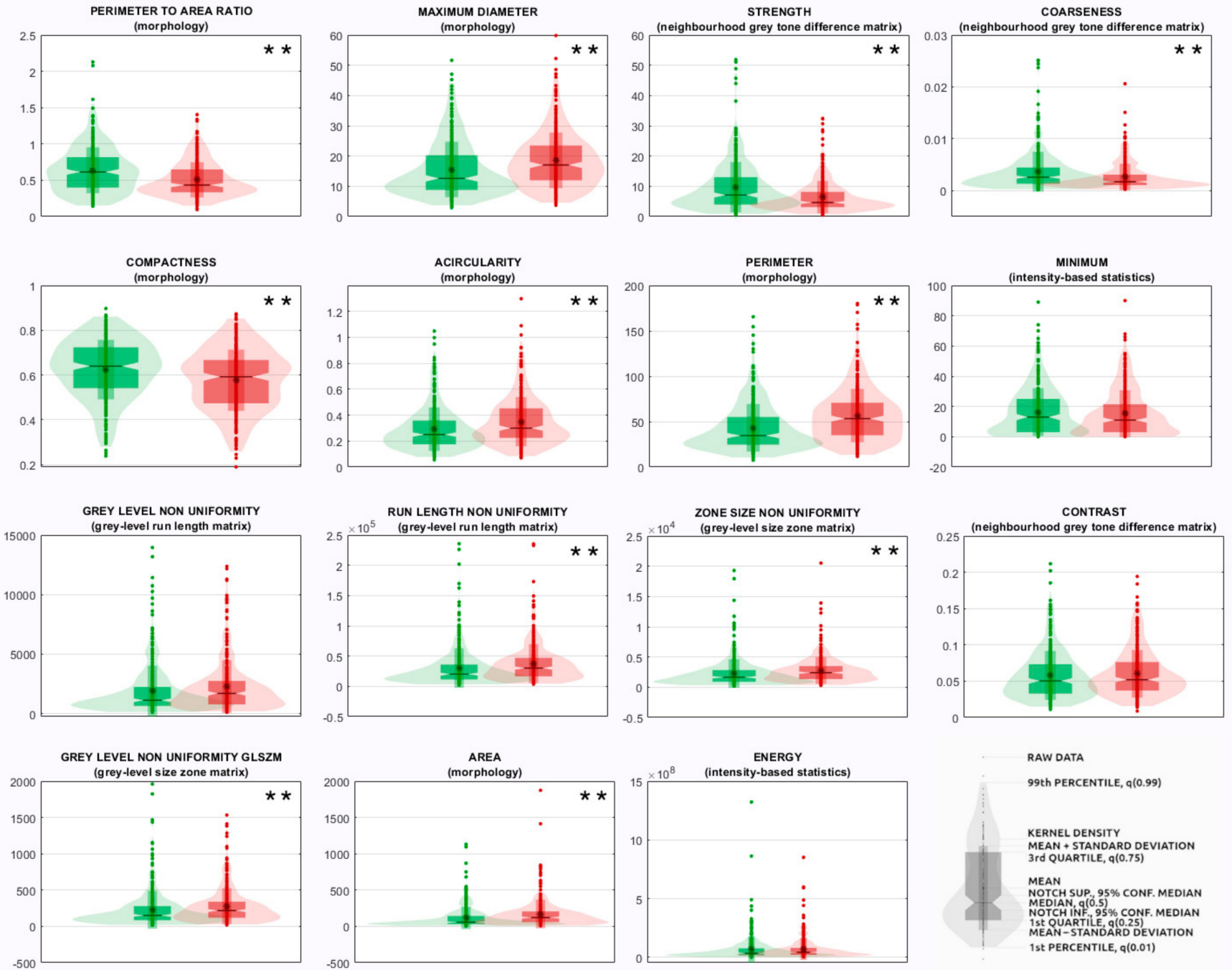

RAW DATA

99th PERCENTILE, a(0.99)

KERNEL DENSITY MEAN + STANDARD DEVIATION 3rd QUARTILE, q(0.75)

MEAN

NOTCH SUP, 95\% CONF. MEDIAN MEDAN, (0.5)
NOTCH INE CONF. MEDIAN
1St OUARTiLE 1SE QUARTiLE, $(0.025)$ MEDTON 1St PERCENTILE, q(0.01)

Figure 2. Ensemble of support vector machines: violin plots and boxplots of the most relevant radiomic predictors ranked from 1 to 15 . Green: benign class. Red: malignant class. ${ }^{* *}$ denotes a statistical significance at 0.005 (adjusted with Bonferroni-Holm correction).

Figures 3 and 4 depict examples of breast masses according to histological diagnosis, as classified by the developed radiomic-based machine learning system. ROIs manually defined by the expert breast radiologist to segment the suspicious lesion are overlapped on the corresponding images. The 107 measured IBSI-compliant radiomic features are reported for these lesions in Table S4.

\subsection{BI-RADS Diagnostic Categories Classification}

Tables 5-7 show the distribution of the BI-RADS categories with respect to histopathology groups as assigned by the ensemble of support vector machines to the breast masses of the Training and internal testing set (598 masses), Testing set I (123 masses), and Testing set II (113 masses). Errors in BI-RADS 3 category assignments by the model were $0.8 \%$ and $2.7 \%$ in Testing set I and Testing set II, respectively.

The certified breast radiologist with 34 years of experience in breast imaging accepted the BI-RADS classes of 114 masses (92.7\%) and modified the BI-RADS classes of 9 breast masses $(7.3 \%)$ (Table S5). In six of nine cases, the model performed better than the radiologist did, since it assigned BI-RADS 3 to masses benign according to histopathology while the radiologist assigned BI-RADS 4 . Two breast masses, malignant according to histopathology, were classified by the model as BI-RADS 4 while the radiologist assigned a BI-RADS 5 classification. These masses were invasive ductal carcinomas according to 
histopathology; thus, the radiologist assigned a more appropriate class of malignancy. The last mass was a granular cell tumor at histopathology, usually considered benign, to whose mass both the model and the radiologist assigned a wrong malignancy BI-RADS class (BI-RADS 4 and 5, respectively); however, we must consider that from a therapeutical point of view, this type of tumor (a rare entity derived from Schwann cells) is aggressive and locally recurrent, therefore requiring surgical excision with curative intent [17].

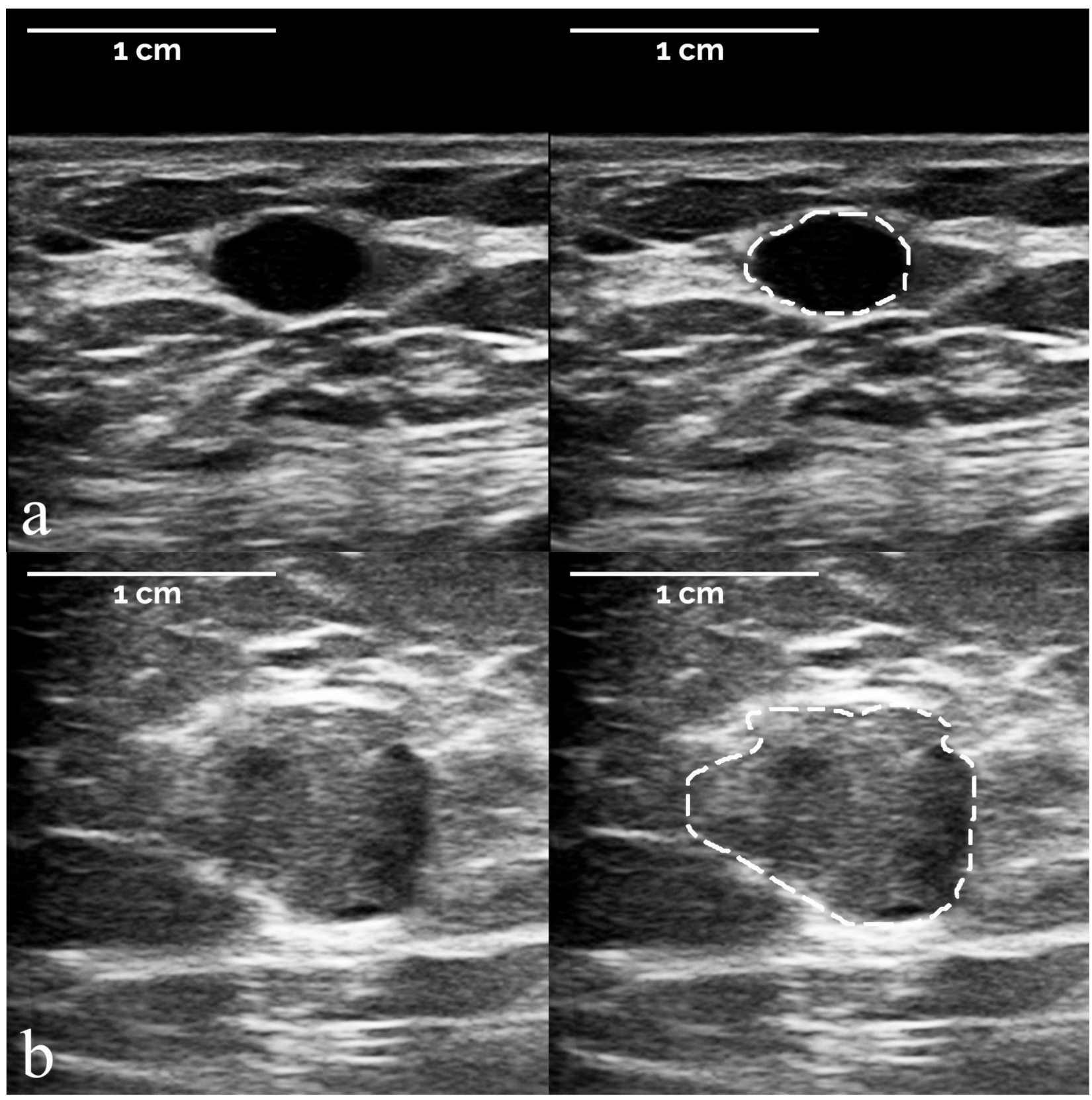

Figure 3. Representative examples of two benign lesions according to histological diagnosis, as classified by the developed radiomic machine learning system. First row (a): true negative (benign lesion correctly classified as $<2 \%$ likelihood of cancer); second row (b): false positive (benign lesion incorrectly classified as $>2 \%$ likelihood of cancer). ROIs were manually defined by the expert breast radiologist to segment the suspicious breast lesion. Radiomic features: (a) compactness 0.807 ; acircularity 0.113 ; center of mass shift 3.495 ; zone size non-uniformity 743.6 ; (b) compactness 0.718 ; acircularity 0.181 ; center of mass shift 4.458; zone size non-uniformity 2255.5. Histopathology: (a) cyst; (b) fibroepithelial proliferation. 
Intra-observer agreement (board-certified breast radiologist with 34 years of experience in breast imaging) in the model classification of BI-RADS was $96 \%(48 / 50)$, with a mean DICE index of $89.7 \% \pm 5.0 \%$. Inter-observer agreement (board-certified breast radiologist with 7 years of experience in breast imaging versus certified breast radiologist with 34 years of experience in breast imaging) in the model classification of BI-RADS was 92\% (46/50), with a mean DICE index of $87.0 \% \pm 9.9 \%$.

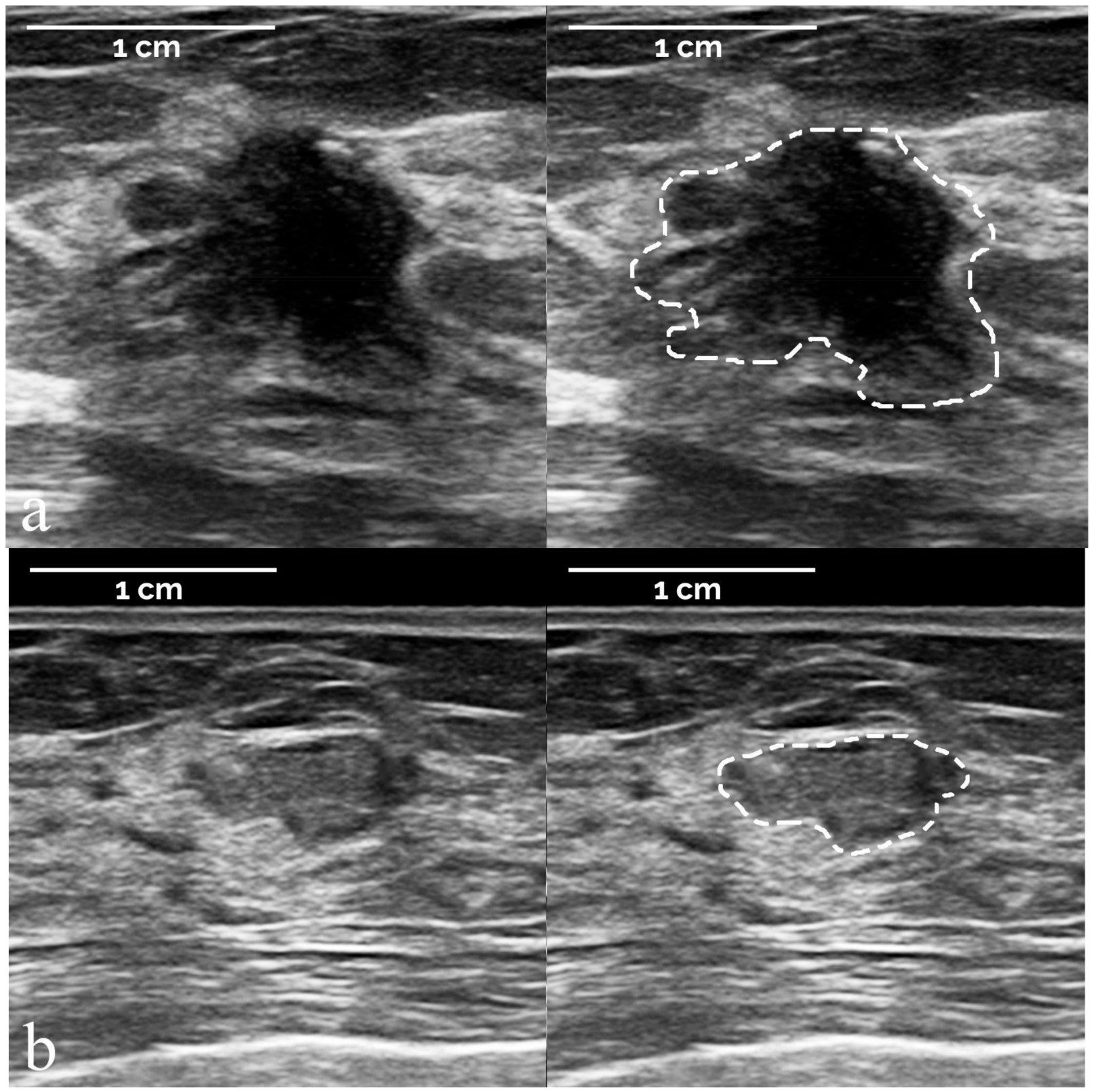

Figure 4. Representative examples of two malignant lesions according to histological diagnosis, as classified by the developed radiomic machine learning system. First row (a): true positive (malignant lesion correctly classified as $>2 \%$ likelihood of cancer); second row (b): false negative (malignant lesion incorrectly classified as $<2 \%$ likelihood of cancer). ROIs were manually defined by the expert breast radiologist to segment the suspicious breast lesion. Radiomic features: (a) compactness 0.569; acircularity 0.326; center of mass shift 3.997; zone size non-uniformity 2600.2; (b) compactness 0.614; acircularity 0.276; center of mass shift 5.861; zone size non-uniformity 2209.0. Histopathology: (a) invasive ductal carcinoma; (b) papillary carcinoma. 
Table 5. Ensemble of support vector machines: BI-RADS diagnostic categories predicted for breast masses of the Training and internal testing set according to histopathology groups.

\begin{tabular}{cccc}
\hline Histopathology Type & BI-RADS 3 (\%) & BI-RADS 4 (\%) & BI-RADS 5 (\%) \\
\hline Fibroadenoma & $8(1.3)$ & $93(15.6)$ & $0(0.0)$ \\
Sclerosing lesions / adenosis & $10(1.7)$ & $37(6.2)$ & $2(0.3)$ \\
Normal breast tissue & $6(1.0)$ & $26(4.3)$ & $1(0.2)$ \\
Inflammatory lesions & $2(0.3)$ & $26(4.3)$ & $0(0.0)$ \\
Papilloma (no atypia) & $1(0.2)$ & $17(2.8)$ & $0(0.0)$ \\
Cysts, ductal ectasia, or seromas & $10(1.7)$ & $13(2.2)$ & $0(0.0)$ \\
Usual ductal hyperplasia & $2(0.3)$ & $9(1.5)$ & $0(0.0)$ \\
Atypical ductal hyperplasia & $0(0.0)$ & $0(0.0)$ & $0(0.0)$ \\
Fibroadenomatoid changes & $2(0.3)$ & $13(2.2)$ & $0(0.0)$ \\
Other benign findings & $8(1.3)$ & $93(15.6)$ & $0(0.0)$ \\
\hline Invasive ductal carcinoma & $5(0.8)$ & $211(35.3)$ & $6(1.0)$ \\
Invasive lobular carcinoma & $2(0.3)$ & $32(5.4)$ & $1(0.2)$ \\
Ductal carcinoma in situ & $4(0.7)$ & $10(1.7)$ & $0(0.0)$ \\
Other malignancies originating & $2(0.3)$ & $24(4.0)$ & $0(0.0)$ \\
from breast tissues & & & $1(0.2)$ \\
Other malignancies (metastases & $0(0.0)$ & $1(0.2)$ & \\
from non breast tissues) & & &
\end{tabular}

Table 6. Ensemble of support vector machines: BI-RADS diagnostic categories predicted for breast masses of the Testing set I according to histopathology groups.

\begin{tabular}{cccc}
\hline Histopathology Type & BI-RADS 3 (\%) & BI-RADS 4 (\%) & BI-RADS 5 (\%) \\
\hline Fibroadenoma & $2(1.6)$ & $19(15.4)$ & $0(0.0)$ \\
Sclerosing lesions / adenosis & $1(0.8)$ & $5(4.1)$ & $0(0.0)$ \\
Normal breast tissue & $0(0.0)$ & $3(2.4)$ & $0(0.0)$ \\
Inflammatory lesions & $2(1.6)$ & $4(3.3)$ & $0(0.0)$ \\
Papilloma (no atypia) & $2(1.6)$ & $4(3.3)$ & $0(0.0)$ \\
Cysts, ductal ectasia, or seromas & $2(1.6)$ & $6(4.9)$ & $0(0.0)$ \\
Usual ductal hyperplasia & $0(0.0)$ & $4(3.3)$ & $0(0.0)$ \\
Atypical ductal hyperplasia & $3(2.4)$ & $5(4.1)$ & $0(0.0)$ \\
Fibroadenomatoid changes & $0(0.0)$ & $1(0.8)$ & $0(0.0)$ \\
Other benign findings & $1(0.8)$ & $8(6.5)$ & $0(0.0)$ \\
\hline Invasive ductal carcinoma & $1(0.8)$ & $40(32.5)$ & $0(0.0)$ \\
Invasive lobular carcinoma & $0(0.0)$ & $3(2.4)$ & $0(0.0)$ \\
Ductal carcinoma in situ & $0(0.0)$ & $2(1.6)$ & $0(0.0)$ \\
Other malignancies originating & $0(0.0)$ & $4(3.3)$ & $0(0.0)$ \\
from breast tissues & & & $0(0.0)$ \\
Other malignancies (metastases & $0(0.0)$ & $1(0.8)$ & \\
from non breast tissues) & & &
\end{tabular}

Table 7. Ensemble of support vector machines: BI-RADS diagnostic categories predicted for breast masses of the Testing set II according to histopathology groups.

\begin{tabular}{cccc}
\hline Histopathology Type & BI-RADS 3 (\%) & BI-RADS 4 (\%) & BI-RADS 5 (\%) \\
\hline Fibroadenoma & $1(0.9)$ & $23(20.4)$ & $0(0.0)$ \\
Sclerosing lesions / adenosis & $2(1.8)$ & $7(6.2)$ & $0(0.0)$ \\
Normal breast tissue & $0(0.0)$ & $2(1.8)$ & $0(0.0)$ \\
Inflammatory lesions & $1(0.9)$ & $1(0.9)$ & $0(0.0)$ \\
Papilloma (no atypia) & $1(0.9)$ & $2(1.8)$ & $0(0.0)$ \\
Cysts, ductal ectasia, or seromas & $1(0.9)$ & $5(4.4)$ & $0(0.0)$ \\
Usual ductal hyperplasia & $1(0.9)$ & $1(0.9)$ & $0(0.0)$ \\
Atypical ductal hyperplasia & $0(0.0)$ & $0(0.0)$ & $0(0.0)$ \\
Fibroadenomatoid changes & $1(0.9)$ & $6(5.3)$ & $0(0.0)$ \\
Other benign findings & $1(0.9)$ & $3(2.7)$ & $0(0.0)$ \\
\hline
\end{tabular}


Table 7. Cont.

\begin{tabular}{cccc}
\hline Histopathology Type & BI-RADS 3 (\%) & BI-RADS 4 (\%) & BI-RADS 5 (\%) \\
\hline Invasive ductal carcinoma & $1(0.0)$ & $40(35.4)$ & $0(0.0)$ \\
Invasive lobular carcinoma & $0(0.0)$ & $4(3.5)$ & $0(0.0)$ \\
Ductal carcinoma in situ & $1(0.9)$ & $2(1.8)$ & $0(0.0)$ \\
Other malignancies originating & $1(0.9)$ & $4(3.5)$ & $0(0.0)$ \\
$\quad$ from breast tissues & $0(0.0)$ & $1(0.9)$ & $0(0.0)$ \\
$\begin{array}{c}\text { Other malignancies (metastases } \\
\text { from non breast tissues) }\end{array}$ & & & \\
\hline
\end{tabular}

\section{Discussion}

In this study, we described the development and validation of a radiomic-based machine learning model aimed at predicting the BI-RADS category and reducing the biopsy rate of ultrasound-detected suspicious breast masses, using a series of 821 images of 834 suspicious breast lesions from 819 patients referred to ultrasound-guided core needle biopsy. Of note, the dataset is characterized by a nearly balanced 1.06:1.0 benign-tomalignant ratio according to histopathology, indicating a high level of expertise in lesion selection, already avoiding the biopsy of a large number of benign lesions. The distribution of the histopathology types was expected, considering that lesion selection was based on ultrasound detection, with a very high proportion, among malignancies, of invasive ductal carcinomas (over three quarters), as already reported in similar series [18,19].

Three ensembles of machine learning supervised classifiers were trained using a balanced image set of 299 benign and 299 malignant lesions. The ensemble of support vector machines, based on a qualified majority vote of over $80 \%$ for predicting the benign nature of the suspicious masses, showed an over 94\% sensitivity (BI-RADS 4-5), allowing to avoid more than 15-18\% of biopsies of benign lesions (BI-RADS 3). Interestingly, these performances remained substantially stable when transitioning from internal cross-validation to two external validation sets, with an over $96 \%$ sensitivity on images from different ultrasound systems from the same vendor (Training and internal testing set and Testing set I).

The ability of individual radiomic features to discriminate malignant from benign masses deserves some comments in relation to the classic BI-RADS descriptors [5,20]. This is a crucial point in terms of explainability to radiologists (and patients as well) of the machine learning model output.

The selected radiomic predictors are able to capture shape, margins, and ultrasonographic pattern of suspicious masses consistently with BI-RADS ultrasound descriptors. Morphological predictors such as compactness and acircularity quantify the deviation of the lesion area from a representative ellipse and circle, respectively, thus being able to distinguish oval and round shape from irregular shapes, the latter more frequent for malignant masses.

The higher values of the center of mass shift predictor in malignant lesions highlight the more asymmetric spatial distribution of intensities for these lesions. These aspects fit well with findings previously reported by Fleury and Marcomini [21], who noted how lesion shape and margins emerged as the most promising BI-RADS features in distinguishing between benign and malignant lesions.

Several texture predictors showed higher values for malignant than for benign lesions, expressing echo-pattern heterogeneity (captured by different non-uniformity features obtained from different texture matrices, i.e., busyness, zone size non-uniformity, grey-level non-uniformity glszm, and dependence count non-uniformity). In addition, the higher values of the texture features coarseness and strength for benign lesions express the tendency for more homogeneous ultrasonographic textural patterns as indicated by BI-RADS descriptors [5].

Less than 1\% of masses were wrongly categorized as BI-RADS 3 in the external Testing set I, less than 3\% in the external Testing set II. Moreover, of the 123 breast lesions of the external Testing set I, 114 (92.7\%) were categorized in the same class by both the model and the expert radiologist, thus showing the possibility of using the tool as an "expert" second 
opinion. Of note, considering the nine disagreement cases, the model assigned the correct benign class to six masses, confirming its potential in reducing the biopsy rate of benign masses. The remaining three masses were classified by both the model and the expert radiologist as positive cases, with BI-RADS 4 given by the model, and BI-RADS 5 given by the radiologist, resulting in the same clinical effect, i.e., referral to biopsy. Two were invasive ductal carcinomas, not needing comments. The other was instead a rare entity (a granular cell tumor, usually considered benign but deserving surgery [17]) that can be considered an "expected" false positive case.

It is important to take into consideration the design of this study, which included only ultrasound-detected breast lesions that underwent ultrasound-guided core needle biopsy. In other words, the large number of lesions considered frankly benign at a qualitative observation by the breast radiologists, i.e., those judged to be associated with null likelihood of cancer (BI-RADS 2, mainly being well-circumscribed anechoic cysts or nonmodified homogeneously hypoechoic fibroadenomas, both of them with regular margins and frequently also posterior enhancement) did not enter this model training dataset. In addition, this series included both symptomatic and asymptomatic breast masses (as common for consecutive series of ultrasound-detected breast masses in real-world clinical practice), the former having a larger size than the latter. This is mirrored by morphological differences between malignant and benign lesions captured by predictors-such as the maximum diameter, perimeter, and area-found to be larger for the malignant lesions than for benign lesions, reflecting this real-world clinical practice scenario.

In order to validate the clinical utility of our model, its diagnostic performances must be contextualized in the clinical decision-making of "to biopsy or not to biopsy" a lesion detected at breast ultrasound. This decision should take into account the high incidence of breast cancer in the female population (in advanced countries, one out of every nine women experiences a breast cancer diagnosis during her lifetime [22-24]) and the increase in cancer probability due to the ultrasound detection of a suspicious lesion, as shown by the experience of targeted ultrasound of mammography-detected [25-27] or MRI-detected lesions [28]. Regarding the inherently high probability of malignancy, we should consider that, in the original consecutive series considered in this work, 451 of 941 lesions (47.9\%) were malignant, and that this rate was substantially maintained after technical exclusions due to not sure lesion identification (404 of 834,48.4\%). This context gives a relevant clinical value to the only apparently low specificity $(15-18 \%)$ provided by our machine learning model, which was still able to maintain an over $94-98 \%$ sensitivity. These results measure the potential clinical advantage of the model, meaning the avoidance of about 1 of 6 biopsies of benign lesions even in this selected series (with about 50\% of malignancies). Notably, all the machine learning model specificity represents a net gain when compared with the $0 \%$ radiologists' specificity (Table 3 ), obliged by the study design, including only biopsied lesions.

In a recent work [29], a commercially available artificial intelligence system based on artificial neural networks was used to evaluate ultrasound-detected breast lesions (classified according BI-RADS categories, from 2 to 5), obtaining a $98 \%$ sensitivity, a $97 \%$ NPV, and a $64 \%$ PPV. Their series was not limited to biopsied lesions only (as was in ours), and the inclusion also of frankly benign lesions (BI-RADS 2) intrinsically increased the specificity of human readers (and of any machine learning model). Indeed, as already observed for diagnostic studies applying breast MRI [30], when considering series solely comprising lesions with histopathology as reference standard, the specificity obviously results to be relatively low, because the benign lesions were suspected to be cancer at a degree to deserve biopsy.

This context can also be further understood considering four large-scale series of breast needle biopsies including 3054 [31], 2420 [32], 20,001 [33], and 22,297 lesions [34], for a total of 47,772 lesions. The proportions of benign lesions were $54.8 \%, 44.3 \%, 51.5 \%$, and $72.6 \%$, respectively, the last series showing that there is no trend in favor of the reduction of the biopsies of benign lesions. Thus, any tool working in this direction is welcome to clinical 
practice and could be used as a second opinion for clinical decision-making in favor of six-month follow-up (as per the BI-RADS 3 diagnostic category, which was introduced with the aim of avoiding biopsy of too many benign lesions) instead of immediate needle biopsy (as per BI-RADS diagnostic categories 4a or higher [5]). Of course, this possibility, occurring in a real-world clinical scenario, should be sustained by a top-level sensitivity (such as the one achieved by our model) combined with an overall BI-RADS 3 NPV ideally higher than $98 \%$, yielding less than $2 \%$ false negative BI-RADS 3 lesions, as recommended by the BI-RADS guidelines [5]. Of note, the NPVs of our model are lower than $98 \%$ (78.3\%, $92.9 \%$, and $75.0 \%$ for the Training and internal testing set, the external Testing set $I$, and the external Testing set II, respectively), but it regards only on BI-RADS 3 lesions, which were all referred to needle biopsy, since our series did not include BI-RADS 3 lesions sent to six-month follow-up. These follow-up cases should have been added to have the overall BI-RADS 3 NPV.

To better clarify the value of our results, we should consider the breast cancer epidemiology at large. According to the International Agency for Research on Cancer [35], in 2020, a total of 2,261,419 new breast cancers were diagnosed worldwide. We can consider that the average rate of benign lesions reported by the four aforementioned large series [31-34] is 29,235 of $47,772(61.1 \%)$, rounded to $60 \%$ (meaning a $40 \%$ malignancy rate), and that the majority of breast needle biopsies are performed under ultrasound guidance (with at least a $70 \%$ estimate $[2,18,36,37])$. Even applying a tool providing only a $15 \%$ additional specificity, we could already save about 356,000 biopsies, i.e., $15 \%$ of the $2,375,000$ needle biopsies of benign lesions performed worldwide under ultrasound guidance every year.

The value of our tool could be much greater when used in conjunction with the physician's evaluation. There are indeed already some studies that demonstrate an increase in physician performance when the decision whether to perform a biopsy or refer to followup is made with the support of decision systems based on AI models predicting the risk of malignancy of a lesion. For example, in the experience reported by Zhao et al. [38], the feasibility of a deep learning-based computer-aided diagnosis (CAD) system was explored in order to improve the diagnostic accuracy of residents in detecting BI-RADS 4a lesions. The authors evaluated the improvement obtained by downgrading BI-RADS 4a lesions identified by radiologists to possibly benign lesions as per CAD prediction. The sensitivity of the integrated results remained at a relatively high level $(>92.7 \%)$, while the specificities of all residents significantly improved after using the results of CAD, rising from $19.5 \%-48.7 \%$ to $46.0 \%-76.1 \%$. Similarly, Barinov et al. [39] showed that through simple fusion schemes, they could increase performance beyond that of either their CAD system or the radiologist alone, obtaining an absolute average PPV increase of $\sim 15 \%$ while keeping original radiologists' sensitivity. Especially less-experienced radiologists could benefit from a CAD system for the diagnosis of ultrasound-detected breast masses, as shown by Lee et al. [40], who compared the evaluation of 500 lesions performed by five experienced and five inexperienced radiologists, with and without CAD; the diagnostic performance of the inexperienced group after combination with CAD result was significantly improved (ROC-AUC: $0.71 ; 95 \%$ CI: 0.65-0.77) compared with the diagnostic performance without CAD (ROC-AUC: 0.65 ; 95\% CI: 0.58-0.71).

However, we should also consider that the final decision to biopsy or to follow-up an ultrasound-detected breast lesion also depends on factors other than ultrasound image characteristics, i.e., on family and personal history of the patient (including the absence of presence of symptoms), and the possible preceding lesion detection on other imaging techniques such as mammography/tomosynthesis or MRI. In this study, we did not take into consideration these different indications to breast ultrasound. In addition, also the patient's psychological condition plays a relevant role in the final decision-making. From this viewpoint, the improvement of clinical decision-making that can be obtained using our model could be estimated in a prospective clinical study and/or in a retrospective reader study, where BI-RADS classes are assigned by our model (based on the consensus of votes expressed by the support vector classifiers of the best ensemble) and then proposed 
to physicians (e.g., the highest consensus for malignancy leads to the highest likelihood of cancer, i.e., BI-RADS 5). Regarding the role of the BI-RADS 3 category in this study, we highlight that here we considered only lesions that underwent needle biopsy, not those that were sent to follow-up and finally downgraded to BI-RADS 2 (for example, for reduction in size), with no possibility to obtain histopathology reference standard. Hence, the potential benefit of the AI tool system could be explored in followed-up lesions with final benign outcome, to assess the role of the model in this specific setting.

A limitation of this study is related to the origin of its patient cohort (a University Hospital located in Northern Italy), which is therefore composed of lesions observed in European Caucasian subjects. While the ultrasound appearance of benign and malignant lesions should not be different in other ethnicities, the different structure of the breast (e.g., Asian women have breasts denser than those of Caucasian women [41-43]) could influence the relation between the lesion and the surrounding tissue: an isoechoic lesion surrounded by fat may be a hypoechoic area surrounded by gland parenchyma. However, considering that our model takes into consideration absolute and not relative signal intensities, we do not expect different performances. A further consideration concerns the choice, adopted in this work, of classical machine learning methods combined with handcrafted image features. We did not consider using a deep learning approach, although it could improve our results and avoid manual segmentation of the masses, because we aimed to provide clinicians with image predictors easy explainable and interpretable with respect to BI-RADS semantic predictors.

In conclusion, in this study, a specifically developed machine learning model based on radiomics to predict the malignant or benign nature of ultrasound-detected suspicious breast lesions was first trained and cross-validated on 598 images of pathology-proven benign or malignant lesions, then underwent independent external validation on 236 other images. Such a model was proven to be effective in predicting BI-RADS 3, 4, and 5 classes and potentially clinically useful in providing an over $15 \%$ reduction of the biopsy rate of lesions finally revealed as benign, while still warranting very high sensitivity. This system can be used in a clinical context as a decision support system to support radiologists in the assignment of BI-RADS classes and toward decision-making regarding short-interval follow-up versus tissue sampling for suspicious breast lesions.

Supplementary Materials: The following are available online at https:/ / www.mdpi.com/article / 10.3390/diagnostics12010187/s1, Table S1: Ensemble of random forest classifiers. Classification performance and statistical significance with respect to chance/random classification ( $p$-value). Performances are reported for a majority vote of $50 \%$ and for the internal testing, Table S2: Ensembles of support vector machine classifiers. Classification performances and statistical significance with respect to chance/random classification ( $p$-value). Performances are reported for a majority vote of $50 \%$ and for the internal testing, Table S3: Ensembles of k-nearest neighbor classifiers. Classification performances and statistical significance with respect to chance/random classification ( $p$-value). Performances are reported for a majority vote of $50 \%$ and for the internal testing, Table S4: Complete list of 107 radiomic features with the values of the four representative lesions (two benign and two malignant) shown in Figures 3 and 4, Table S5: BI-RADS classes assigned by the ensemble of support vector machines (AI model) and the certified breast radiologist, Figure S1: Violin plots and boxplots of the most relevant features ranked from 16 to 25 .

Author Contributions: Conceptualization, C.S., I.C. and F.S.; data curation, M.I., C.S., V.M., G.C., E.S., A.C., S.S. and L.A.C.; formal analysis, M.I., C.S., E.S. and I.C.; funding acquisition, C.S., I.C. and F.S.; investigation, M.I., C.S., V.M., G.C., E.S., A.C., S.S. and L.A.C.; methodology, M.I., C.S., A.C., I.C. and F.S.; project administration, C.S. and I.C.; resources, C.S., V.M., E.S., S.S., L.A.C., I.C. and F.S.; software, M.I., C.S., G.C., E.S. and I.C.; supervision, S.S., L.A.C., I.C. and F.S.; validation, M.I., C.S., V.M., A.C., I.C. and F.S.; visualization, M.I., G.C., E.S. and A.C.; writing-original draft, M.I., G.C., I.C. and F.S.; writing-review and editing, M.I., C.S., V.M., G.C., E.S., A.C., S.S., L.A.C., I.C. and F.S. All authors have read and agreed to the published version of the manuscript.

Funding: The Authors acknowledge support for this research from the European Union's HORIZON 2020 research and innovation program (CHAIMELEON project, grant agreement \#952172). 
Institutional Review Board Statement: The study was conducted according to the guidelines of the Declaration of Helsinki, and approved by the Ethics Committee of IRCCS Ospedale San Raffaele (protocol code "SenoRetro", first approved on 9 November 2017, then amended on 18 July 2019, and on 12 May 2021).

Informed Consent Statement: Patient consent was waived due to the retrospective nature of the study.

Data Availability Statement: All data analyzed for this study are presented in the manuscript or in the supplementary material.

Conflicts of Interest: Christian Salvatore declares to be CEO of DeepTrace Technologies S.R.L., a spinoff of Scuola Universitaria Superiore IUSS, Pavia, Italy. Matteo Interlenghi and Isabella Castiglioni declare to own DeepTrace Technologies S.R.L shares. Simone Schiaffino declares to have received travel support from Bracco Imaging, and to be a member of the speakers' bureau for General Electric Healthcare. Francesco Sardanelli declares to have received grants from, or to be member of, the speakers' bureau/advisory board for Bayer Healthcare, the Bracco Group, and General Electric Healthcare. All other authors have nothing to disclose.

\section{References}

1. Evans, A.; Trimboli, R.M.; Athanasiou, A.; Balleyguier, C.; Baltzer, P.A.; Bick, U.; Camps Herrero, J.; Clauser, P.; Colin, C.; Cornford, E.; et al. Breast ultrasound: Recommendations for information to women and referring physicians by the European Society of Breast Imaging. Insights Imaging 2018, 9, 449-461. [CrossRef]

2. Bick, U.; Trimboli, R.M.; Athanasiou, A.; Balleyguier, C.; Baltzer, P.A.T.; Bernathova, M.; Borbély, K.; Brkljacic, B.; Carbonaro, L.A.; Clauser, P.; et al. Image-guided breast biopsy and localisation: Recommendations for information to women and referring physicians by the European Society of Breast Imaging. Insights Imaging 2020, 11, 12. [CrossRef]

3. Tomkovich, K.R. Interventional Radiology in the Diagnosis and Treatment of Diseases of the Breast: A Historical Review and Future Perspective Based on Currently Available Techniques. Am. J. Roentgenol. 2014, 203, 725-733. [CrossRef] [PubMed]

4. Biganzoli, L.; Marotti, L.; Hart, C.D.; Cataliotti, L.; Cutuli, B.; Kühn, T.; Mansel, R.E.; Ponti, A.; Poortmans, P.; Regitnig, P.; et al Quality indicators in breast cancer care: An update from the EUSOMA working group. Eur. J. Cancer 2017, 86, 59-81. [CrossRef]

5. D'Orsi, C.J.; Sickles, E.A.; Mendelson, E.B.; Morris, E.A. ACR BI-RADS ${ }^{\circledR}$ Atlas, Breast Imaging Reporting and Data System, 5th ed.; American College of Radiology: Reston, VA, USA, 2013.

6. Castiglioni, I.; Rundo, L.; Codari, M.; Di Leo, G.; Salvatore, C.; Interlenghi, M.; Gallivanone, F.; Cozzi, A.; D’Amico, N.C.; Sardanelli, F. AI applications to medical images: From machine learning to deep learning. Phys. Med. 2021, 83, 9-24. [CrossRef] [PubMed]

7. Tagliafico, A.S.; Piana, M.; Schenone, D.; Lai, R.; Massone, A.M.; Houssami, N. Overview of radiomics in breast cancer diagnosis and prognostication. Breast 2020, 49, 74-80. [CrossRef] [PubMed]

8. Lee, S.-H.; Park, H.; Ko, E.S. Radiomics in Breast Imaging from Techniques to Clinical Applications: A Review. Korean J. Radiol. 2020, 21, 779-792. [CrossRef] [PubMed]

9. Bitencourt, A.; Daimiel Naranjo, I.; Lo Gullo, R.; Rossi Saccarelli, C.; Pinker, K. AI-enhanced breast imaging: Where are we and where are we heading? Eur. J. Radiol. 2021, 142, 109882. [CrossRef]

10. Hu, Q.; Giger, M.L. Clinical Artificial Intelligence Applications: Breast Imaging. Radiol. Clin. N. Am. 2021, 59, 1027-1043. [CrossRef] [PubMed]

11. Stavros, A.T.; Thickman, D.; Rapp, C.L.; Dennis, M.A.; Parker, S.H.; Sisney, G.A. Solid breast nodules: Use of sonography to distinguish between benign and malignant lesions. Radiology 1995, 196, 123-134. [CrossRef]

12. Zwanenburg, A.; Vallières, M.; Abdalah, M.A.; Aerts, H.J.W.L.; Andrearczyk, V.; Apte, A.; Ashrafinia, S.; Bakas, S.; Beukinga, R.J.; Boellaard, R.; et al. The Image Biomarker Standardization Initiative: Standardized Quantitative Radiomics for High-Throughput Image-based Phenotyping. Radiology 2020, 295, 328-338. [CrossRef] [PubMed]

13. TRACE4. Available online: http:/ / www.deeptracetech.com/temp/TechnicalSheet_TRACE4.pdf (accessed on 7 January 2022).

14. Koo, T.K.; Li, M.Y. A Guideline of Selecting and Reporting Intraclass Correlation Coefficients for Reliability Research. J. Chiropr. Med. 2016, 15, 155-163. [CrossRef] [PubMed]

15. Mann, H.B.; Whitney, D.R. On a Test of Whether one of Two Random Variables is Stochastically Larger than the Other. Ann. Math Stat. 1947, 18, 50-60. [CrossRef]

16. Holm, S. A Simple Sequentially Rejective Multiple Test Procedure. Scand. J. Stat. 1979, 6, 65-70.

17. Meani, F.; Di Lascio, S.; Wandschneider, W.; Montagna, G.; Vitale, V.; Zehbe, S.; Harder, Y.; Parvex, S.L.; Spina, P.; Canonica, C.; et al. Granular cell tumor of the breast: A multidisciplinary challenge. Crit. Rev. Oncol. Hematol. 2019, 144, 102828. [CrossRef]

18. Van Breest Smallenburg, V.; Nederend, J.; Voogd, A.C.; Coebergh, J.W.W.; van Beek, M.; Jansen, F.H.; Louwman, W.J.; Duijm, L.E.M. Trends in breast biopsies for abnormalities detected at screening mammography: A population-based study in the Netherlands. Br. J. Cancer 2013, 109, 242-248. [CrossRef] 
19. Allison, K.H.; Abraham, L.A.; Weaver, D.L.; Tosteson, A.N.A.; Nelson, H.D.; Onega, T.; Geller, B.M.; Kerlikowske, K.; Carney, P.A.; Ichikawa, L.E.; et al. Trends in breast biopsy pathology diagnoses among women undergoing mammography in the United States: A report from the Breast Cancer Surveillance Consortium. Cancer 2015, 121, 1369-1378. [CrossRef]

20. Mendelson, E.B.; Böhm-Vélez, M.; Berg, W.A. ACR BI-RADS ${ }^{\circledR}$ Ultrasound. In ACR BI-RADS ${ }^{\circledR}$ Atlas, Breast Imaging Reporting and Data System; American College of Radiology: Reston, VA, USA, 2013.

21. Fleury, E.; Marcomini, K. Performance of machine learning software to classify breast lesions using BI-RADS radiomic features on ultrasound images. Eur. Radiol. Exp. 2019, 3, 34. [CrossRef] [PubMed]

22. DeSantis, C.E.; Ma, J.; Gaudet, M.M.; Newman, L.A.; Miller, K.D.; Goding Sauer, A.; Jemal, A.; Siegel, R.L. Breast cancer statistics, 2019. CA Cancer J. Clin. 2019, 69, 438-451. [CrossRef] [PubMed]

23. Torre, L.A.; Islami, F.; Siegel, R.L.; Ward, E.M.; Jemal, A. Global Cancer in Women: Burden and Trends. Cancer Epidemiol. Biomark. Prev. 2017, 26, 444-457. [CrossRef] [PubMed]

24. La Vecchia, C.; Carioli, G. The epidemiology of breast cancer, a summary overview. Epidemiol. Biostat. Public Health 2018, 15, e12853-1-e12853-2. [CrossRef]

25. Flobbe, K.; Bosch, A.M.; Kessels, A.G.H.; Beets, G.L.; Nelemans, P.J.; von Meyenfeldt, M.F.; van Engelshoven, J.M.A. The Additional Diagnostic Value of Ultrasonography in the Diagnosis of Breast Cancer. Arch. Intern. Med. 2003, 163, 1194. [CrossRef]

26. McCavert, M.; O'Donnell, M.E.; Aroori, S.; Badger, S.A.; Sharif, M.A.; Crothers, J.G.; Spence, R.A.J. Ultrasound is a useful adjunct to mammography in the assessment of breast tumours in all patients. Int. J. Clin. Pract. 2009, 63, 1589-1594. [CrossRef] [PubMed]

27. Guo, R.; Lu, G.; Qin, B.; Fei, B. Ultrasound Imaging Technologies for Breast Cancer Detection and Management: A Review. Ultrasound Med. Biol. 2018, 44, 37-70. [CrossRef]

28. Spick, C.; Baltzer, P.A.T. Diagnostic Utility of Second-Look US for Breast Lesions Identified at MR Imaging: Systematic Review and Meta-Analysis. Radiology 2014, 273, 401-409. [CrossRef]

29. Mango, V.L.; Sun, M.; Wynn, R.T.; Ha, R. Should We Ignore, Follow, or Biopsy? Impact of Artificial Intelligence Decision Support on Breast Ultrasound Lesion Assessment. Am. J. Roentgenol. 2020, 214, 1445-1452. [CrossRef]

30. Baltzer, P.A.T.; Sardanelli, F. The Mantra about Low Specificity of Breast MRI. In Breast MRI for High-Risk Screening; Sardanelli, F., Podo, F., Eds.; Springer International Publishing: Cham, Switzerland, 2020; pp. 11-21.

31. Andreu, F.J.; Sáez, A.; Sentís, M.; Rey, M.; Fernández, S.; Dinarès, C.; Tortajada, L.; Ganau, S.; Palomar, G. Breast core biopsy reporting categories-An internal validation in a series of 3054 consecutive lesions. Breast 2007, 16, 94-101. [CrossRef] [PubMed]

32. Youk, J.H.; Kim, E.-K.; Kim, M.J.; Oh, K.K. Sonographically Guided 14-Gauge Core Needle Biopsy of Breast Masses: A Review of 2420 Cases with Long-Term Follow-Up. Am. J. Roentgenol. 2008, 190, 202-207. [CrossRef]

33. El-Sayed, M.E.; Rakha, E.A.; Reed, J.; Lee, A.H.; Evans, A.J.; Ellis, I.O. Audit of performance of needle core biopsy diagnoses of screen detected breast lesions. Eur. J. Cancer 2008, 44, 2580-2586. [CrossRef] [PubMed]

34. Jung, I.; Han, K.; Kim, M.J.; Moon, H.J.; Yoon, J.H.; Park, V.Y.; Kim, E.-K. Annual Trends in Ultrasonography-Guided 14-Gauge Core Needle Biopsy for Breast Lesions. Korean J. Radiol. 2020, 21, 259-267. [CrossRef] [PubMed]

35. Sung, H.; Ferlay, J.; Siegel, R.L.; Laversanne, M.; Soerjomataram, I.; Jemal, A.; Bray, F. Global Cancer Statistics 2020: GLOBOCAN Estimates of Incidence and Mortality Worldwide for 36 Cancers in 185 Countries. CA Cancer J. Clin. 2021, 71, 209-249. [CrossRef] [PubMed]

36. Helbich, T.H.; Matzek, W.; Fuchsjäger, M.H. Stereotactic and ultrasound-guided breast biopsy. Eur. Radiol. 2004, 14, 383-393. [CrossRef]

37. O'Flynn, E.A.M.; Wilson, A.R.M.; Michell, M.J. Image-guided breast biopsy: State-of-the-art. Clin. Radiol. 2010, 65, 259-270. [CrossRef] [PubMed]

38. Zhao, C.; Xiao, M.; Liu, H.; Wang, M.; Wang, H.; Zhang, J.; Jiang, Y.; Zhu, Q. Reducing the number of unnecessary biopsies of USBI-RADS 4a lesions through a deep learning method for residents-in-training: A cross-sectional study. BMJ Open 2020, 10, e035757. [CrossRef] [PubMed]

39. Barinov, L.; Jairaj, A.; Paster, L.; Hulbert, W.; Mammone, R.; Podilchuk, C. Decision quality support in diagnostic breast ultrasound through Artificial Intelligence. In Proceedings of the 2016 IEEE Signal Processing in Medicine and Biology Symposium (SPMB), Philadelphia, PA, USA, 3 December 2016; pp. 1-4.

40. Lee, J.; Kim, S.; Kang, B.J.; Kim, S.H.; Park, G.E. Evaluation of the effect of computer aided diagnosis system on breast ultrasound for inexperienced radiologists in describing and determining breast lesions. Med. Ultrason. 2019, 21, 239-245. [CrossRef] [PubMed]

41. del Carmen, M.G.; Halpern, E.F.; Kopans, D.B.; Moy, B.; Moore, R.H.; Goss, P.E.; Hughes, K.S. Mammographic Breast Density and Race. Am. J. Roentgenol. 2007, 188, 1147-1150. [CrossRef] [PubMed]

42. Bae, J.-M.; Kim, E.H. Breast Density and Risk of Breast Cancer in Asian Women: A Meta-analysis of Observational Studies. J. Prev. Med. Public Health 2016, 49, 367-375. [CrossRef]

43. Rajaram, N.; Mariapun, S.; Eriksson, M.; Tapia, J.; Kwan, P.Y.; Ho, W.K.; Harun, F.; Rahmat, K.; Czene, K.; Taib, N.A.M.; et al. Differences in mammographic density between Asian and Caucasian populations: A comparative analysis. Breast Cancer Res. Treat. 2017, 161, 353-362. [CrossRef] 